\title{
Nanomilling for Aberration - Corrected TEM and HAADF STEM
}

\author{
R. R. Cerchiara, * P. E. Fischione,* J. Liu*, J. M Matesa*, A. C. Robins*, H. L. Fraser** and \\ A. Genc** \\ * E. A. Fischione Instruments, Inc., 9003 Corporate Circle, Export, PA 15632 \\ ** The Ohio State University, Columbus, $\mathrm{OH} 43210$
}

Nanomilling as a complementary technique to conventional or focused ion beam (FIB) milling has been demonstrated for in situ lift-out samples of ceramics and certain metallic alloys. [1,2] Damaged layers (2-5 nm thick) may remain after final FIB milling with $2-5 \mathrm{keV} \mathrm{Ga}^{+}$ions. [3] These layers contain amorphous sample material and implanted $\mathrm{Ga}+$, with the distribution dependent upon the sample material and preparation protocol. Unless the damaged layer thickness can be reduced or eliminated, artifacts will occur during imaging in the transmission electron microscope (TEM).

For this research, a concentrated argon ion beam (50 to $2000 \mathrm{eV})$ contained in a beam diameter of 2 - $4 \mu \mathrm{m}$ is directed at low incident angles $\left(0\right.$ to $\left.\pm 10^{\circ}\right)$ to a sample maintained at a temperature between $25^{\circ}$ and $-175^{\circ} \mathrm{C}$. The damaged layer is removed without altering the complex sample chemistry or crystalline structure. Re-deposition during nanomilling is minimized by careful orientation of the lamella with respect to its support grid, Fig. 1. Typical lamella dimensions are $\sim 5$ $\mu \mathrm{m}$ X $10 \mu \mathrm{m}$ X $100 \mathrm{~nm}$. Because the lamella and ion beam are of the same relative size, there is no $\mathrm{Ar}^{+}$ion contact with the support grid. Imaging occurs as the electrons released during the sputtering process are collected by the secondary electron detector (SED). Once an image is scanned, selective targeting of the area of interest occurs, and the final ion milling protocol is executed.

The nanomilling rate varies as a function of the sample material and instrument parameters. Data was extracted from atomic force microscopy (AFM) measurements made of trenches ion milled into single crystal $\mathrm{Si}$. The maximum achievable rate was $7.7 \mathrm{~nm} / \mathrm{min}$. Improvements in 200kV HRTEM image quality were observed for $\mathrm{Si}$ when the results obtained after milling with $\mathrm{Ga}^{+}$ions and subsequent milling with $\mathrm{Ar}^{+}$ions were compared, Fig. 2 (left and center). [4] Atomic resolution imaging of the dumbbell structure was possible as depicted in Fig. 2 (right). [5]

Nanomilling reduced the damage in materials that were environmentally sensitive and / or highly ordered. This was demonstrated in the case of the $\mathrm{Ar}^{+}$broad ion beam (BIB) milled Ti alloy depicted in Fig. 3 (left). Additional results will be reviewed for titanates based on strontium and barium. Aberration - corrected, high angle annular dark field (HAADF) 200kV STEM images of a $\mathrm{SrTiO}_{3}$ sample after FIB and subsequent nanomilling are also shown in Fig. 3.

\section{References}

[1] P. E. Fischione et al., Presentation CL4, FEMMS, Kasteel Vaalsbroek, Holland, 2005.

[2] A. Genc et al., Microsc. Microanal. 13 (Suppl. 2), 2007, pp.1520 - 1521.

[3] L. A. Giannuzzi et al., Microsc. Microanal. 11 (Suppl. 2), 2005, pp. $828-829$.

[4] Uncorrected images courtesy of P. Midgley, D. Cooper and R. E. Dunin-Borkowski, University of Cambridge, U.K.

[5] Corrected image courtesy of A. Kirkland and C. Hetherington, University of Oxford, U.K. 


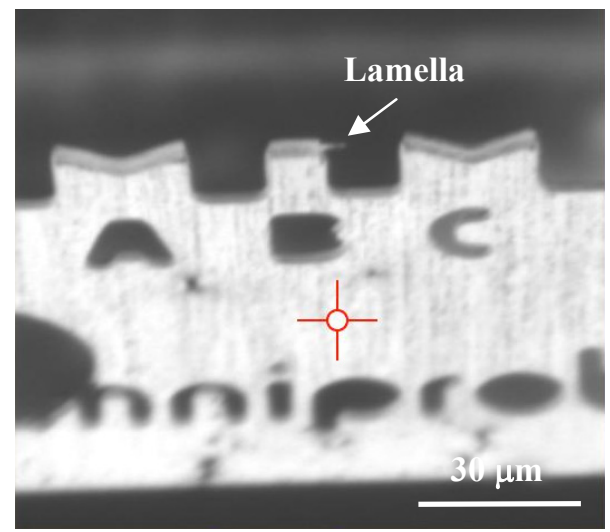

Fig. 1: $500 \mathrm{eV}$ secondary electron image of a lamella acquired using the NanoMill at $15^{\circ}$ stage tilt.
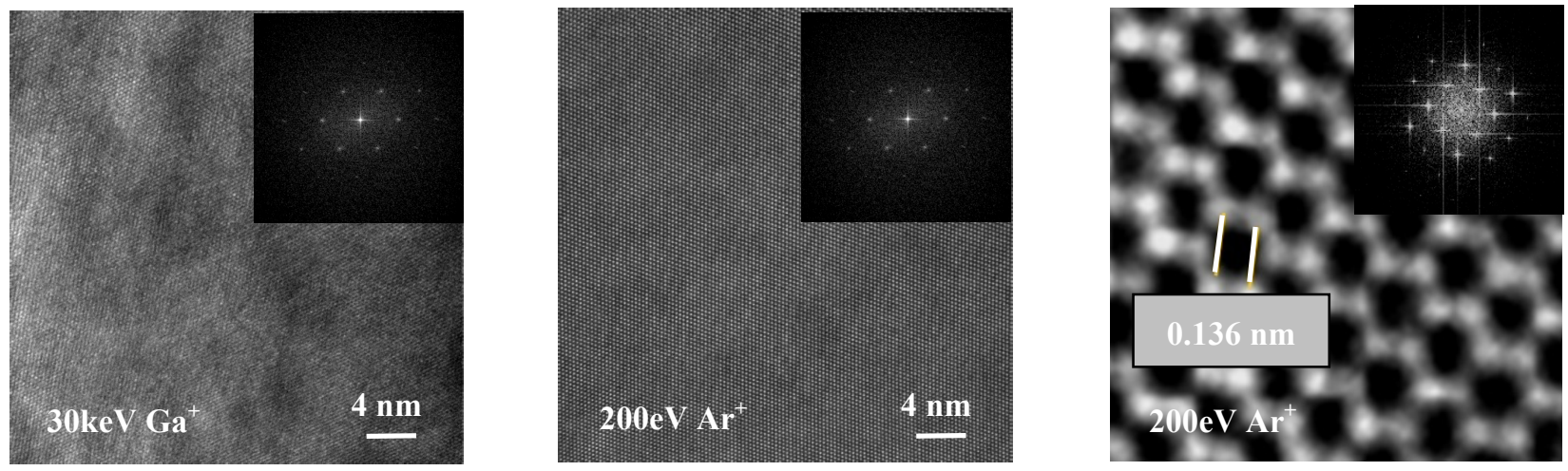

Fig. 2. Uncorrected HRTEM images of Si after $30 \mathrm{keV}$ FIB (left) and subsequent $200 \mathrm{eV}$ nanomilling (center). Aberration - corrected image (right) showing dumbbell structure as evidenced by the FFT.
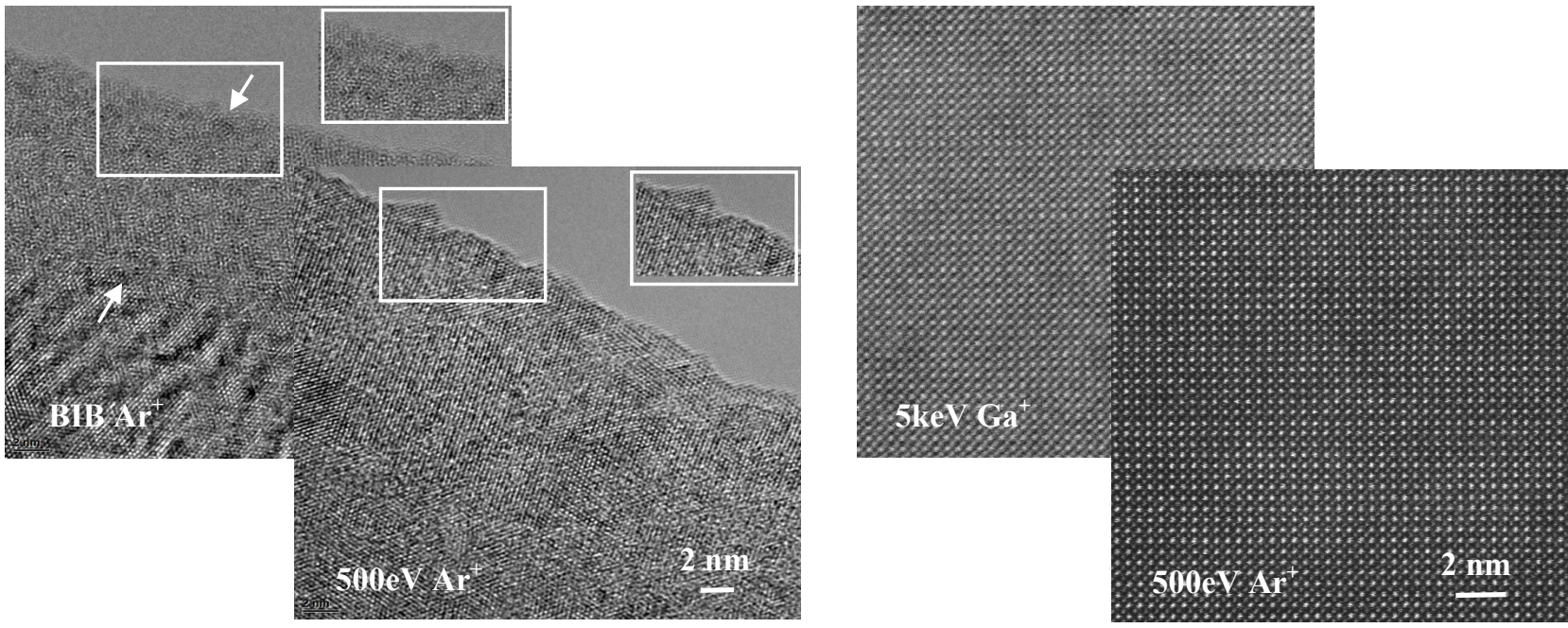

Fig. 3. Uncorrected HRTEM images of a Ti alloy after $\mathrm{Ar}^{+} \mathrm{BIB}$ milling and subsequent $500 \mathrm{eV}$ nanomilling (left). The $10-12 \mathrm{~nm}$ wide amorphous rim was removed. Aberration - corrected HAADF STEM images of $\mathrm{SrTiO}_{3}$ after $5 \mathrm{keV}$ FIB and subsequent $500 \mathrm{eV}$ nanomilling (right). 